\title{
Lipiodol remnants outside the abdominal cavity
}

\author{
Chieko Kurimoto $^{1}$, Shunji Goto ${ }^{1}$, Yoshikazu Yamagishi ${ }^{2}$, Fumiko Chiba $^{3}$, Hirotaro Iwase ${ }^{2}$, \\ and Eiji Kato ${ }^{1}$ \\ ${ }^{1}$ JCHO Funabashi Central Hospital \\ ${ }^{2}$ Chiba University Graduate School of Medicine School of Medicine \\ ${ }^{3}$ The University of Tokyo Graduate School of Medicine Faculty of Medicine
}

July 29, 2021

\begin{abstract}
We experienced a case of pregnancy after hysterosalpingogram and residual lipiodol in the extraperitoneal space. Initially, we suspected a metallic remnant, however, analysis by mass spectrometer confirmed that it was a remnant of lipiodol.
\end{abstract}

Title

Lipiodol remnants misinterpreted as a metal device on postoperative abdominal X-ray images

Authors

Chieko Kurimoto $^{1}$, Shunji Goto ${ }^{1}$, Yoshikazu Yamagishi ${ }^{2},{\text { Fumiko } \text { Chiba }^{2,3} \text {, Hirotaro } \text { Iwase }^{2,3} \text {, Eiji Kato }}^{1}$

${ }^{1}$ Department of Obstetrics, Funabashi Central Hospital

${ }^{2}$ Laboratory of Toxicology and Environmental Health, Graduate School of Pharmaceutical Sciences and Department of Legal Medicine, Graduate School of Medicine, Chiba University

${ }^{3}$ Department of Forensic Medicine, Graduate School of Medicine, The University of Tokyo

\section{Corresponding author}

Chieko Kurimoto

Department of Obsterics, Funabashi Central Hospital

6-13-10, Kaijin, Funabashi-shi, Chiba 273-8556, Japan

E-mail: ckurimoto06@yahoo.co.jp

Telephone number: +81-47-433-2111, FAX number: +81-47-435-2655

\section{Key Words}

Lipiodol, hysterosalpingogram

\section{Main Text}

A 36-year-old nulliparous woman with 40 weeks of gestation was admitted to our department due to premature rupture of the membranes. The hysterosalpingogram had been performed with $4 \mathrm{ml}$ of lipiodol approximately 10 months prior to her presentation, and the fallopian tube was normal. An emergency cesarean section was performed as intrauterine infection was suspected. The child's condition at birth was uneventful. Postoperative abdominal x-ray showed high enhancement shadows (Fig.1). It was confirmed 
that no gauze and instrument was lost at the end of the surgery. Abdominal CT images showed high density shadows outside the abdominal cavity along the inguinal canal (Fig.2), suggesting the presence of a metal-device. The surgical removed object was a cystic mass about $2 \mathrm{~cm}$ in diameter with a yellow liquid. Mass-spectrometry showed the substance was highly consistent with the same lot number of lipiodol used at the previous clinic. There are a few case reports of intraperitoneal remnants of lipiodol (1-2). In our case, it was found outside the abdominal cavity. Gynecologists should keep in mind the possibility of contrast agent remnants when examining X-ray images on the patient with a history of hysterosalpingogram.

\section{Author contribution}

CK: managed the patient, collected the data and wrote the paper.

SG: managed the patient and wrote the paper.

YY: collected the data, performed the analysis.

FC: collected the data, performed the analysis.

HI: collected the data, performed the analysis.

EK: managed the patient and wrote the report.

\section{Conflicts of Interest and Source of Funding}

The authors declare no conflicts of interest associated with this manuscript.

\section{Ethical approval and Acknowledgments}

We greatly thank Yuichi Kodaira for English language editing, and our hospital's medical staff for the assistance. We would like to acknowledge the patient for providing consent to share her case.

\section{References}

1. Wakabayashi Y, Hashimura N, Kubouchi T. Retained Lipiodized Oil Misdiagnosed as residual metallic material. Radiation Medicine 2004; 22(5): 362-363.

2. Miyamoto Y, Tsujimoto T, Iwai K, Ishida K, Uchimoto R, Miyazawa T, Azuma H. Safety and pharmacokinetics of iotrolan in hysterosalpingography. Retention and irritability compared with Lipiodol. Invest Radiol 1995; 30(9): 538-543. 


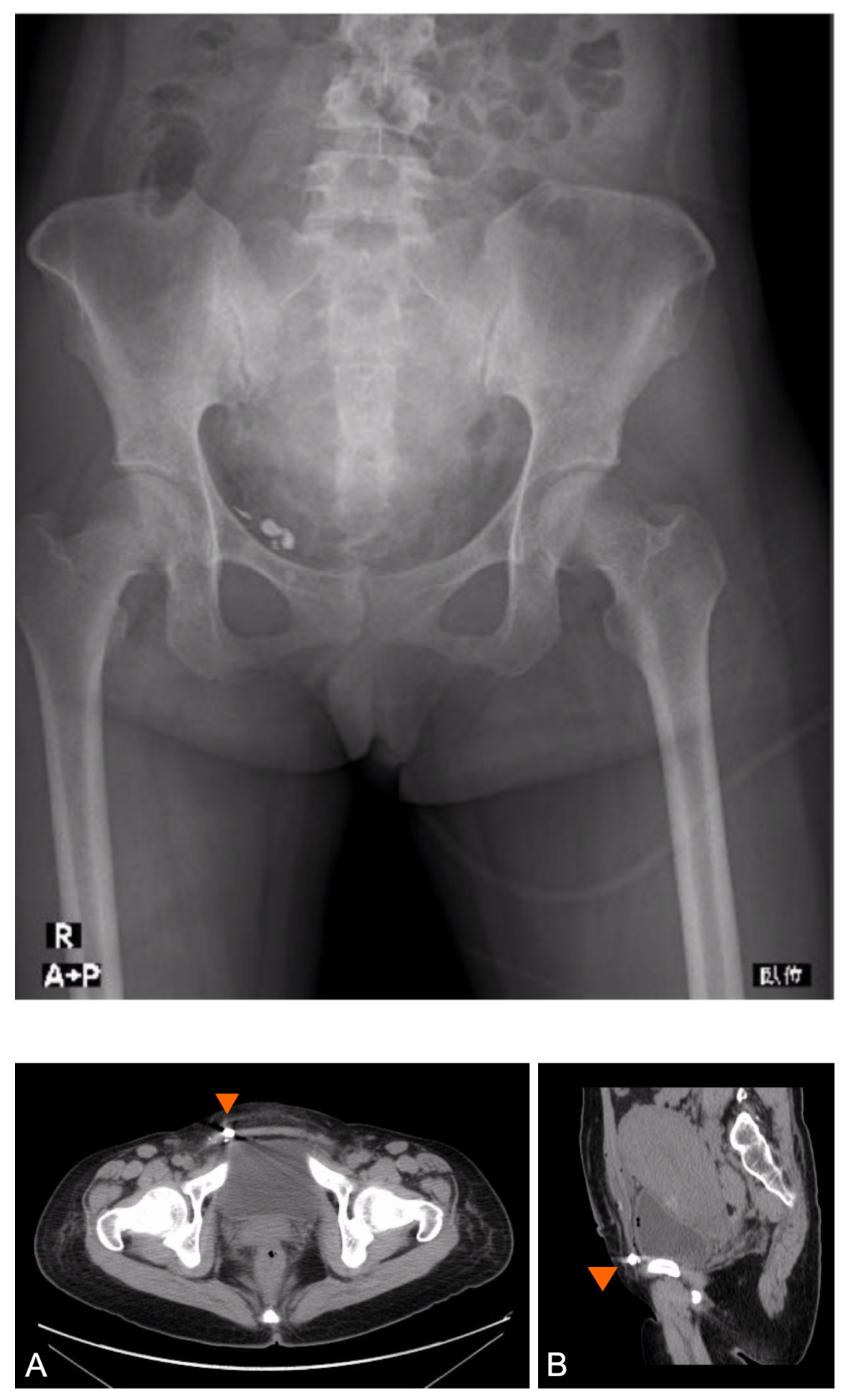

